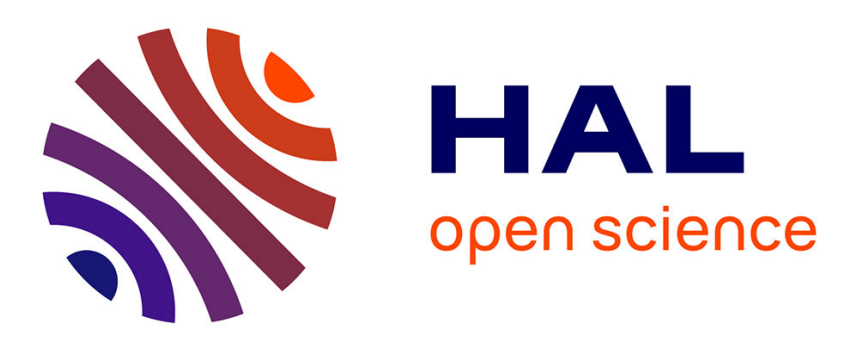

\title{
Auger recombination and multiple exciton generation in colloidal two-dimensional perovskite nanoplatelets: Implications for light-emitting devices
}

Carolina Villamil Franco, Benoit Mahler, Christian Cornaggia, Thomas Gustavsson, Elsa Cassette

\section{To cite this version:}

Carolina Villamil Franco, Benoit Mahler, Christian Cornaggia, Thomas Gustavsson, Elsa Cassette. Auger recombination and multiple exciton generation in colloidal two-dimensional perovskite nanoplatelets: Implications for light-emitting devices. ACS Applied Nano Materials, 2021, 4 (1), pp.558-567. 10.1021/acsanm.0c02868 . hal-03175975

\section{HAL Id: hal-03175975 \\ https://hal.science/hal-03175975}

Submitted on 5 Oct 2021

HAL is a multi-disciplinary open access archive for the deposit and dissemination of scientific research documents, whether they are published or not. The documents may come from teaching and research institutions in France or abroad, or from public or private research centers.
L'archive ouverte pluridisciplinaire HAL, est destinée au dépôt et à la diffusion de documents scientifiques de niveau recherche, publiés ou non, émanant des établissements d'enseignement et de recherche français ou étrangers, des laboratoires publics ou privés. 


\section{Auger Recombination And Multiple Exciton Generation In Colloidal Two-Dimensional Perovskite}

\section{Nanoplatelets: Implications For Light-Emitting Devices}

AUTHOR NAMES. Carolina Villamil Franco ${ }^{1}$, Benoît Mahler ${ }^{2}$, Christian Cornaggial, Thomas Gustavsson ${ }^{1}$ and Elsa Cassette ${ }^{1, *}$

AUTHOR ADDRESS. 'Iniversité Paris-Saclay, CEA, CNRS, Laboratoire Interactions, Dynamiques et Lasers (LIDYL), 91191 Gif-sur-Yvette, France. ${ }^{2}$ Université de Lyon, Université Claude Bernard Lyon 1, CNRS, Institut Lumière Matière (ILM), F-69622 Villeurbanne, France.

KEYWORDS. Halide perovskite, quantum confinement, Auger recombination, multiple exciton generation, 2D nanoplatelets 


\section{INTRODUCTION}

By combining strong photoluminescence, color purity and enhanced stability with easy and cheap solution-processing fabrication, two-dimensional (2D) halide perovskites have taken a central role in the development of electroluminescent diodes (LEDs), laser gain media or singlephoton sources. ${ }^{1-3}$ For all these various light-emitting devices, understanding the excitonexciton annihilation process, which ultimately limits the exciton density in these materials, is essential in order to control the device performances. For example, lasing threshold and LED efficiency are affected by the rapid non-radiative Auger recombination (AR) rate that limits the maximum electron-hole pair density in the photoactive material. ${ }^{4}$ It is well established that AR is the dominant recombination process that occurs in semiconductor nanostructures under highexcitation density. 5,6

Over the last few years, AR has been the subject of several investigations in weakly-confined colloidal perovskite nanocrystals $(\mathrm{NCs}){ }^{7-9}$ However, studies in strongly confined 2D perovskite samples are limited to a few cases: methylammonium lead iodide (MAPI) 2D layered thin films ${ }^{10,11}$ and 5 monolayers (MLs) $\mathrm{CsPbBr}_{3}$ nanoplatelets (NPLs). ${ }^{12,13}$ While an increase of the AR rate is expected with the increase of the Coulomb interactions in confined systems, a surprisingly slow "bimolecular" AR rate of a few $10^{-4}$ and $10^{-3} \mathrm{~cm}^{2} / \mathrm{s}$ was reported in $2 \mathrm{D}$ perovskite films in the strong confinement regime (1 to $5 \mathrm{MLs}$ ), leading to effective $\mathrm{AR}$ times on the order of hundreds of picoseconds to nanoseconds. ${ }^{10,11}$ Interestingly, in a recent publication a decrease from 78 ps to 18 ps was reported in the effective biexcitonic recombination time of weakly-confined $\mathrm{CsPbI}_{3} \mathrm{NCs}$ (size $12.1 \mathrm{~nm}$ ) compared to in morestrongly confined ones $(6.2 \mathrm{~nm}) .{ }^{14}$ Depending on the nature of the photoexcited species (free charge carrier or excitons), the AR process will follow third-or second-order kinetics. ${ }^{15,16}$ Besides, the anisotropic geometry of a system like the 2D quantum wells can induce further 
modifications in the measured kinetics such as asymmetric exciton-exciton interactions ${ }^{17}$ and diffusion-limited recombination. ${ }^{11,18}$

Here, we studied the multiple exciton dynamics in strongly-confined lead iodide colloidal NPLs of 2 and 3 MLs by varying the excitation fluence from less than one to tens of $\mu \mathrm{J} / \mathrm{cm}^{2}$. We found a fast intrinsic AR rate with a corresponding effective recombination time of less than $10 \mathrm{ps}$ at high exciton density $\left(>5 \times 10^{11} \mathrm{~cm}^{-2}\right)$ while exciton diffusion slows down the AR process to hundreds of ps when the initial exciton density is low.

We also studied the formation of multiple excitons in these NPL samples by observing the AR that occurs after high photon energy and low fluence excitation. So far, multiple exciton generation (MEG) has only been observed in weakly-confined iodide-based perovskite nanocrystals, $\mathrm{CsPb}_{3}$, and $\mathrm{FAPbI}_{3}$, using transient absorption spectroscopy. ${ }^{14,19,20}$ These measurements are always very sensitive to photo-charging effects and/or a misinterpretation as a result of a too high excitation fluence. ${ }^{21}$ Surprisingly, Klimov and coworkers did not observed any MEG signatures in Cs-based perovskite NCs using time-resolved photoluminescence (TRPL) techniques. ${ }^{7}$ Here, we show that MEG induces a fast AR rate in 3 ML-thick NPLs due to the proximity of the two "geminate" excitons, that can be easily isolated from other nonradiative recombinations.

\section{EXPERIMENTAL SECTION}

Perovskite Nanoplatelets synthesis. All the commercially available chemicals used for the synthesis of colloidal perovskite nanostructures are listed in the Supporting Information (SI). Thin perovskite nanoplatelets were synthesized at room temperature by precipitation by injecting the precursor solution into a non-polar solvent, here the toluene (see full details in the SI). 
Steady-state optical characterization. Absorption measurements were carried out with a UV/Vis Lambda 850 spectrophotometer (Perkin Elmer) covering the 175-900 $\mathrm{nm}$ spectral range. Photoluminescence was carried out with a Fluorolog 3-22 spectrofluorometer (HORIBA JOBIN-YVON) equipped with a R928P photomultiplier tube detector (200-870 nm) and a CW Xenon arc lamp (450 W, 250-2500 nm). A UV enhanced silicon photodiode reference detector monitores and compensates for variation in the Xenon lamp intensity.

Time-correlated single photon counting (TCSPC). We used a mode-locked Ti:Sapphire (MIRA 900, Coherent) pumped by a solid state laser (VERDI V10, Coherent) and providing $120 \mathrm{fs}$ pulses tunable between 720 and $950 \mathrm{~nm}$ (about $2 \mathrm{~W}$ at $800 \mathrm{~nm}$ ), at $76 \mathrm{MHz}$ repetition rate. The rate was reduced to $200 \mathrm{kHz}$ using a pulse picker (Model 9200, Coherent). The output pulse train was subsequently frequency-doubled, providing $400 \mathrm{~nm}$ pulses. The polarization of the excitation beam was controlled by a Fresnel rhomb (EKSMA) and set parallel to the detection. The fluorescence was collected on a monochromator (HR250, Jobin-Yvon). A Schott WG 420 filter was positioned in front of the entrance slit in order to cut the diffused excitation light. The fluorescence was detected with a microchannel plate photomultiplier (R1564 U, Hamamatsu). The temporal resolution is about $60 \mathrm{ps}$ with a time window $<100 \mathrm{~ns}$. To avoid photodegradation, the samples were continuously refreshed using a circulating system and a stirred bar in a $3 \mathrm{~mm}$ quartz cell.

Transient Absorption (TA). All measurements of the colloidal perovskite nanostructures were carried out at room temperature in a $1 \mathrm{~mm}$-thick flow cell connected to a peristaltic pump in order to refresh the sample at the focus position to avoid photodamage and photocharging effects. Flexible and organic solvent compatible F-5500-A fluoroelastomer (Saint-Gobain ${ }^{\circledR}$ ) tubing and Teflon adaptors were used to build the flow system. The employed sample solutions were prepared with optical density (OD) below 0.3 at the excitation wavelength and above in anhydrous toluene (or chloroform). We used a home-built TA fs spectrometer with an initial 
Ti:Sa laser source at $3 \mathrm{kHz}, 40$ fs and about $2 \mathrm{~W}$ to generate a tunable excitation in the 500-650 nm range with a non-collinear optical parametric amplifier (NOPA) and a white-light (WL) continuum for the probe. The output of the NOPA, after a temporal compression involving chirped mirror pair and a double-prism compressor, can be frequency doubled using a set of lens, a BBO crystals and polarizers to filter out the residual visible beam. The temporal resolution is about $100 \mathrm{fs}$ in the visible and about $300 \mathrm{fs}$ for the UV experiments. The maximum pump-probe time delay that can be reached is about $3.2 \mathrm{~ns}$ using a long delay line with a retroreflector mounted on a motorized stage $(60 \mathrm{~cm}$ long, minimum step $200 \mathrm{~nm}$, IMS600CCHA from Newport). The continuum probe beam was filtered to remove the fundamental $800 \mathrm{~nm}$ and reduced in intensity by a neutral density filter. Both pump and probe were set vertical in polarization using broadband half-waveplates. The repetition rate of the pump is reduced to half $(1.5 \mathrm{kHz})$ to block one pulse out of two, allowing for measures with and without the pump.

The continuum spectrum was registered at $3 \mathrm{kHz}$ by a commercial spectrograph (IsoPlane 160, Princeton Instruments) coupled to a CCD camera (ProEM-HS 1024B, Princeton Instruments). To measure a spectrum of 1024 pixels at $3 \mathrm{kHz}$ with the CCD camera (3000 frames/s), we use a fast-custom acquisition mode with a vertical binning of 64 pixels (13 $\mu \mathrm{m}$ each) over the region of interest (ROI) closest to the chip and an exposure time of $1 \mu \mathrm{s}$. The TA signal was calculated as follow: $\Delta A(\lambda, t)=-\log \left(\frac{1}{m} \sum_{k=0}^{m-1} \frac{I_{2 k}^{O N}(\lambda, t)-I_{\text {fluo }}(\lambda)}{I_{2 k+1}^{O F F}(\lambda, t)-I_{\text {dark }}(\lambda)}\right)$, with $I(\lambda, t)$ the WL spectrum measured at a pump-probe time delay $t$ with the pump "ON" for a pulse $2 k$ (even) and the pump OFF for the following pulse $2 k+1$ (odd), $I_{\text {fluo }}$ the spectrum recorded with the probe beam blocked, $I d_{\text {ark }}$ the spectrum measured with both pump and probe blocked and $m$ the number of ON/OFF kinetic cycle pairs to average at one position of the translation state (for each different $t$ ). Both $I_{\text {fluo }}$ and $I_{\text {dark }}$ correspond to background spectra that need to be subtracted before performing the 
ratio of the WL spectrum. They are collected just before the scan collection, averaged over 3000 pulses.

\section{RESULTS AND DISCUSSION}
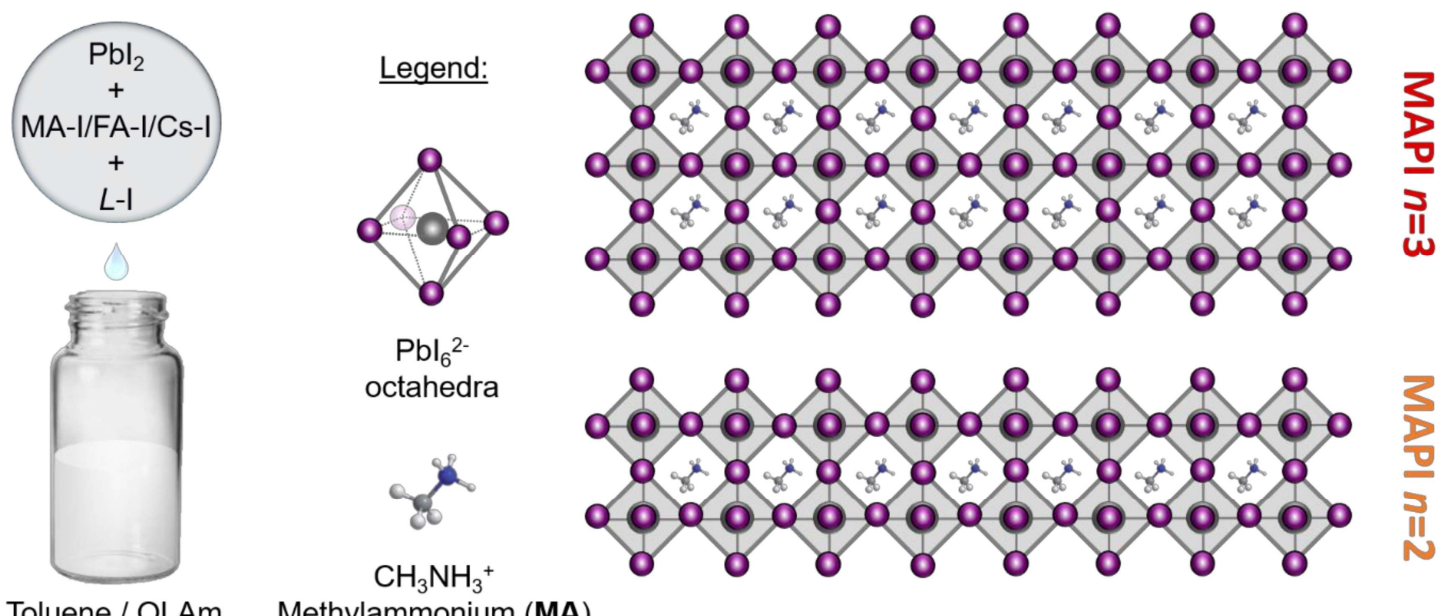

Toluene / OLAm
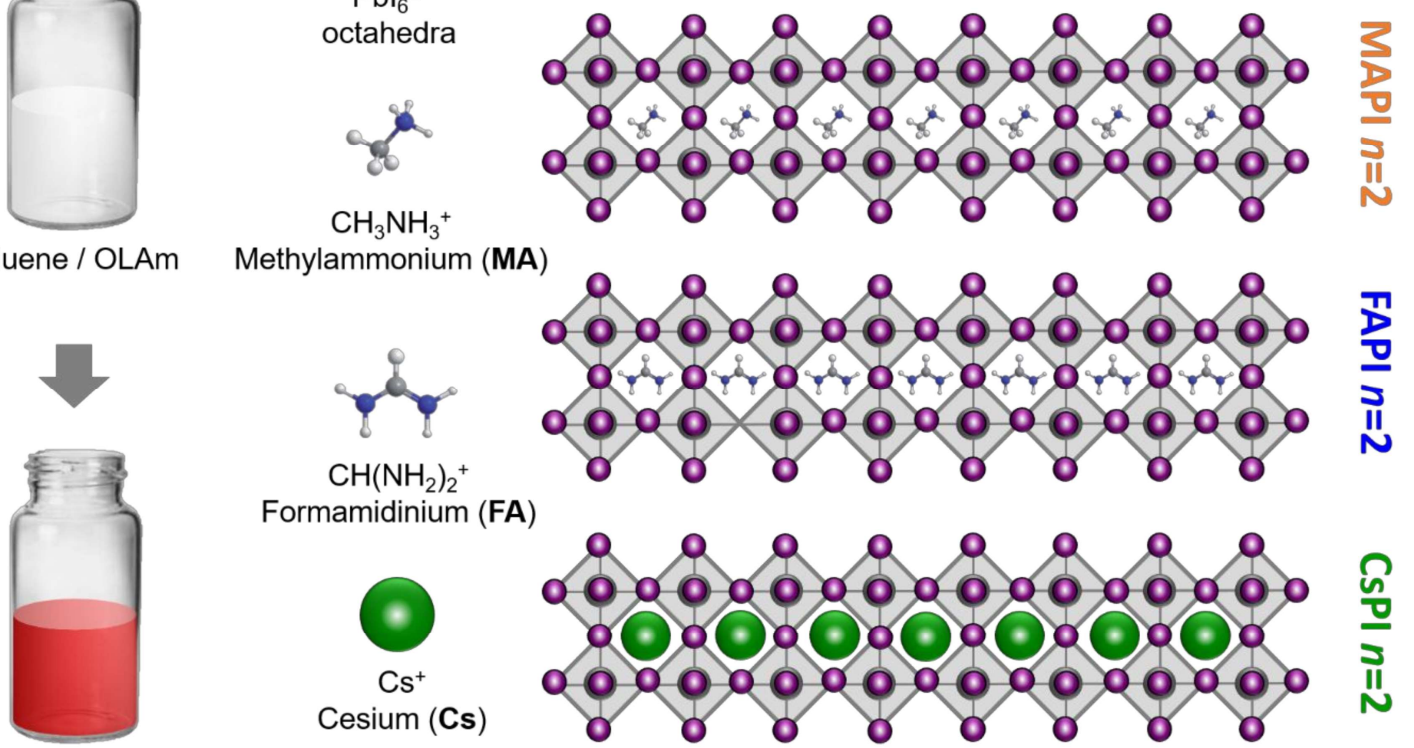

$\mathrm{CH}_{3} \mathrm{NH}_{3}{ }^{+}$

Methylammonium (MA)

Scheme 1. Schematics of the lead iodide perovskite NPL samples and their colloidal synthesis $(L=$ ammonium ligand, OLAm $=$ oleylammine $)$.

Nanoplatelet sample characterizations. Colloidal perovskite NPLs were synthesized at room temperature based on the ligand assisted re-precipitation (LARP) method ${ }^{22}$ (Scheme 1, details in EXPERIMENTAL SECTION and SI). From the initial report of two-monolayer-thick $L_{2}\left[\mathrm{APbI}_{3}\right] \mathrm{PbI}_{4}(n=2 \mathrm{MAPI}, \mathrm{FAPI}$ and $\mathrm{CsPI}) \mathrm{NPLs}$ with $L$, an ammonium ligand and $\mathrm{A}$ an inorganic (cesium, Cs) or organic (methylammonium, MA or formaminidium, FA) cation, we extended the synthesis to three-monolayer-thick $L_{2}\left[\mathrm{MAPbI}_{3}\right]_{2} \mathrm{PbI}_{4}$ NPLs (MAPI $n=3$ ). The 
schematics of the different samples is shown in Scheme 1. Unlike the ligand-assisted exfoliation procedures used in previously reported hybrid colloidal NPLs, ${ }^{23,24}$ the direct solution approach allows a more well-defined population (i.e. number of monolayers) in thinner NPLs.

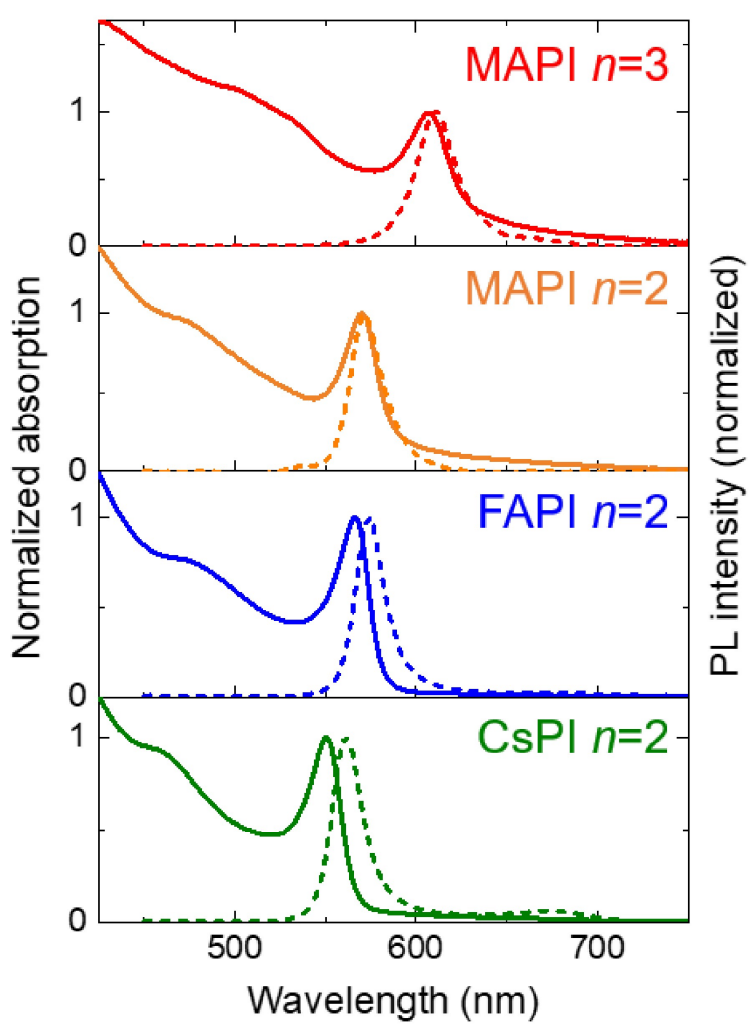

Figure 1. Absorption (full line) and photoluminescence (dashed line) spectra of colloidal perovskite NPLs of two monolayers (CsPI, FAPI, MAPI $n=2)$ and three monolayers (MAPI $n=3)$.

The steady-state absorption and photoluminescence (PL) spectra of the synthesized NPLs dispersed in toluene are shown in Figure 1. For each sample, the energy corresponding to the first exciton transition matches the previous reports of strongly confined 2D NPLs and thin films ${ }^{25-27}$. Except for CsPI $n=2$, all NPL samples present lateral dimensions from hundreds of nanometers to a few micrometers (see SI) and thus confinement in these dimensions can be 
neglected. The CsPI sample, however, might display some degree of confinement in one of the lateral dimensions (nanoribbons with a width of $17 \pm 3 \mathrm{~nm}$ and a length of several hundreds of nm, see Figure S1 in SI). While the small blue shift in the spectrum of Cs-based perovskite samples compared to MA- and FA-based ones is typical, ${ }^{28}$ here it might be the consequence of the extra confinement effects, which could affect the AR rate. ${ }^{12}$

Time resolved photoluminescence experiments. TR-PL is a standard method to study multiexciton dynamics in colloidal nanostructures. Since the PL signal is proportional to the average number of bound electron-hole pairs in the excited nanoparticles, which allows to distinguish different kinetic recombination processes. ${ }^{29}$ Here, we employ the time-correlated single-photon counting (TCSPC) technique (see EXPERIMENTAL SECTION) with an instrument response function (IRF) of about $60 \mathrm{ps}$, to record luminescence decays of the four colloidal perovskite NPLs at the band-edge after excitation at $400 \mathrm{~nm}$. By varying the excitation fluence from 40 $\mathrm{nJ} / \mathrm{cm}^{2}$ to $15 \mu \mathrm{J} / \mathrm{cm}^{2}$, multiexciton interactions may be revealed.
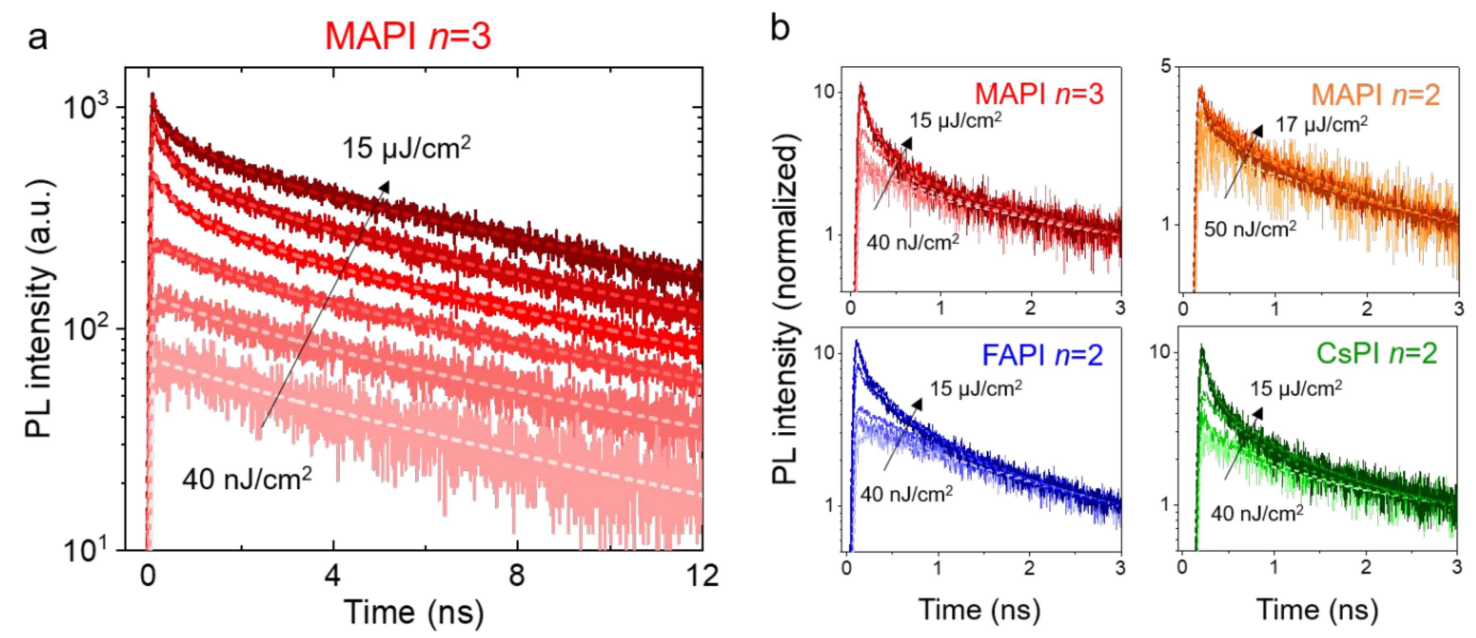

Figure 2. Fluence-dependent PL decay traces of the perovskite NPLs excited at $400 \mathrm{~nm}$ (bandedge detection). (a) Experimental data (full lines) and fit (dashed lines) for MAPI $n=3$ NPLs. (b) Experimental data and fit of the four different NPL samples normalized at long time to highlight the extra decay at early time at high excitation fluence. 
The PL decay traces recorded for MAPI $n=3$ at different excitation fluences at $400 \mathrm{~nm}$ are shown in Figure 2a. The data for the $n=2$ NPL samples are displayed in Figure $\mathbf{S 2}$ in the SI. The PL decay traces could be well fitted by a multiexponential function. All the corresponding fit parameters are given in the SI Tables S1-S4.

At low excitation fluencies, the dynamics are almost mono-exponential for MAPI and FAPI NPLs, but a better description is obtained for all the samples using a biexponential decay function, having time constants $\tau_{2}$ and $\tau_{3}$. Starting at about $2 \mu \mathrm{J} / \mathrm{cm}^{2}$ (see the calculation of the excitation fluences in the SI), an extra short-lived component appears, characterized by a time constant $\tau_{1}$ that decreases from a few hundreds to a few tens of ps with increasing excitation fluence. At the same time, the middle component $\tau_{2}$ decreases from just below (or few ns) to a few hundreds of ps. The long component $\tau_{3}$, however, remains almost constant and is $10 \pm 1$, $5.7 \pm 0.1,3.2 \pm 0.6$ and $5.9 \pm 0.1 \mathrm{~ns}$ for MAPI $n=3$, MAPI $n=2$, FAPI $n=2$, and CsPI $n=2$, respectively.

The constant value of the long component $\tau_{3}$ for all excitation fluences shows that the PL signal detected at several $\mathrm{ns}$ is mainly associated to mono-exciton recombination. Indeed, recombination of free charge carriers will lead to bimolecular recombination, as previously observed in bulk halide perovskites. ${ }^{30}$ The amplitudes associated with this long component saturate much faster than the total amplitudes (i.e. intensity at time $t=0$ ), as seen in Figure S3 in the SI for MAPI $n=3$. This means that while a high excitation fluence can generate several excitons within a single NPL, multiexciton recombinations are efficient enough to reduce the dynamics at long times to single exciton recombination. The larger value of $\tau_{3}$ for the $n=3 \mathrm{NPL}$ sample can be explained by its larger fluorescence quantum yield (see SI).

The consistency of the long time dynamics due to mono-exciton recombination allows us to normalize the PL decay traces at a long time and to isolate the extra faster decay component appearing at larger excitation densities. We assigned this faster component to multiple exciton 
dynamics (Figure 2b), in line with previous reports. ${ }^{8,31-33}$ The decrease of the corresponding effective multiexciton decay times $\tau_{1}$ and $\tau_{2}$ with the initial number of created excitons is expected for quadratic multi-exciton recombination (see discussion below). As these time constants are on the order of hundreds to tens of ps, they correspond to non-radiative AR rather than radiative biexcitonic recombination (see discussion in SI). Here, trion dynamics, which corresponds to another kind of Auger recombination can be neglected since the samples were circulated within the $3 \mathrm{~mm}$-cell and the repetition rate decreased to $200 \mathrm{kHz}$ to prevent from photo-charging and photo-degradation effects. ${ }^{5,34}$

If the main excited species are excitonic, AR follows bimolecular recombination kinetics: ${ }^{15}$

$$
\frac{d \tilde{n}}{d t}=-\beta \tilde{n}(t)^{2}
$$

with $\tilde{n}(t)$ the time-dependent exciton density and $\beta$ the "bimolecular" AR rate. For highly asymmetric 2D nanostructures, the exciton density is surfacic (number of excitons $N$ per NPL surface, in $\mathrm{cm}^{-2}$ ), such as $\beta$ is typically displayed in $\mathrm{cm}^{2} / \mathrm{s}$. We note that a factor $1 / 2$ should normally be added in front of $\beta$ because, for relatively low excitation photon energy, the reexcited exciton is not ionized and relax back to the band-edge at a cooling rate expected much higher than the AR rate. We note however that this factor was not taken into account previously so for a fair comparison we intentionally omit it.

The bimolecular Auger recombination requires a sufficiently high density of excitons to occur since it involves short-range exciton-exciton interactions that are described either by a collision process or a Förster energy transfer which scales as $\left(1 / d_{\mathrm{X}-\mathrm{X}}\right)^{6}$, where $d_{\mathrm{X}-\mathrm{X}}$ is the distance between the excitons (one exciton transfers its energy to re-excite the other exciton). ${ }^{35}$ In the two limiting cases of immobile excitons (e.g. self-trapped excitons $)^{36}$ and large exciton delocalization length in the 2D plane (the wavefunction of the exciton center-of-mass motion extends well beyond $\left.d_{\mathrm{x}-\mathrm{x}}{ }^{2}\right),{ }^{37} \beta$ is constant in time and the solution to the Equation 1 has the analytical form: ${ }^{15}$ 


$$
\tilde{n}(t)=\frac{\tilde{n}(0)}{1+\tilde{n}(0) \beta t}
$$

with $\tilde{n}(0)$, the initial exciton density.

The measured PL intensity is proportional to the averaged instantaneous number of excitons $N(t)$ and thus to the corresponding density $\tilde{n}(t)$. We thus use the Equation 2 to fit the isolated multiexciton PL decay traces, by fixing the initial exciton density $\tilde{n}(0)$ calculated from the excitation fluence and the linear absorption coefficient of the sample (Figure S4a in SI). The early-time TR-PL decay were well reproduced by the fit and we obtained an effective bimolecular AR rate $\beta$ decreasing from $8.2 \times 10^{-2}$ to $1.1 \times 10^{-2} \mathrm{~cm}^{2} / \mathrm{s}$ with increasing excitation fluence from 0.15 to $15 \mu \mathrm{J} / \mathrm{cm}^{2}$. These bimolecular AR rate values, listed in the first column of Table S6 in the SI, are plotted against the exciton density in Figure 3 (black squares). It is here worthwhile to note that the $\beta$ values are orders of magnitude lower when using Equation S2 in the SI to fit the full PL decay, including both multi- and single-exciton dynamics (second column of Table S6 in SI), as reported by Huang and co. and Garrot and co.. ${ }^{10,11}$ However, it is clear from the corresponding fits (Figure S4b), that these values are not representative of the multiexciton dynamics as the early time dynamics are not well reproduced. That is why we found important to fit the isolated multiexciton dynamics to extract the $\beta$ values.

The experimental dynamics become faster when increasing the initial exciton density $\tilde{n}(0)$, which is in agreement with the effective linear AR rates, extracted from the multi-exponential fit, increasing with the excitation fluence (Figure 3, red dots). This expresses a super-linear dependency of the kinetics related to multi-exciton dynamics depending on the exciton density. As expected for a pure bimolecular recombination ( $c f$ Equation 1 and 2), the dynamics indeed become faster when increasing the initial exciton density. However, the corresponding bimolecular AR rate $\beta$ should remain constant. The fact that the experimental $\beta$ values extracted by bimolecular fits using Equation 2, decrease with the initial exciton density $\tilde{n}(0)$, shows that 
the isolated multi-exciton dynamics deviate from bimolecular kinetics. In particular, it shows that the experimental kinetic dependency on $\tilde{n}$ is sub-quadratic.

This trend can be understood if the bimolecular AR rate $\beta$ in Equation 1 is time-dependent. This is for example the case if the bimolecular AR is limited by the exciton diffusion in the 2D plane. In that case, the diffusion process reduces the dependency of the AR kinetics on the exciton density $\tilde{n}$. Indeed, excitons initially separated by distances larger than the typical reaction radius (i.e. moderated $\tilde{n}(0)$ ) will diffuse and eventually encounter, leading to an effective recombination.

Several works on the exciton-exciton annihilation at moderate excitation fluence in molecular systems presenting highly-limited delocalization length have included the random walk motion (diffusion) of the excitons before collision and resulting exciton-exciton recombination, at moderate excitation fluence. ${ }^{11,38}$ For example, in one-dimensional systems such as linear Jaggregates, the bimolecular rate $\beta(t)$ is predicted to have a $t^{-1 / 2}$ dependency. ${ }^{39}$ Excitons in $2 \mathrm{D}$ halide perovskites are expected to be much more spatially delocalized than their counterparts in molecular systems. However, due to the ionic nature of the perovskite structure, excitons in 2D perovskite structures might present reduced delocalization length along the $2 \mathrm{D}$ plane compared to transition metal dichalcogenides $\left(\mathrm{MoS}_{2}, \mathrm{WSe}_{2}\right.$, etc...) or II-VI semiconductors such as CdSe. To the best of our knowledge, the effect of the exciton diffusion on the excitonexciton annihilation process in perovskite nanostructures has only been discussed by Li et al. and Deng et al.. ${ }^{11,12}$ The former study did not investigate the evolution of the AR times with the excitation fluency but extracted the biexciton dynamics from transient absorption measurements on $n=5 \mathrm{CsPbBr}_{3}$ NPLs with different lateral areas and found it very fast but sensitive to the NPL geometry (recombination time: 14 to $70 \mathrm{ps)} .{ }^{12}$ The latter work reported a transient absorption microscopy study of $2 \mathrm{D}$ layered ( $n=1$ to 5 ) perovskite thin films. By 
comparing the diffusion constant and the AR rate extracted from their analysis, the authors concluded that the AR is reaction-limited and not diffusion-limited. ${ }^{11}$

Exponential functions are commonly used to fit the AR dynamics. ${ }^{7-8,12-13}$ In the absence of accurate analytical formula to describe the Equation 1 with time-dependent bimolecular AR rate, we found that the multiexponential fit was adapted to effectively fit the decay traces with high quality. In order to relate the AR rate in $\mathrm{s}^{-1}$ extracted from the multiexponential fit with

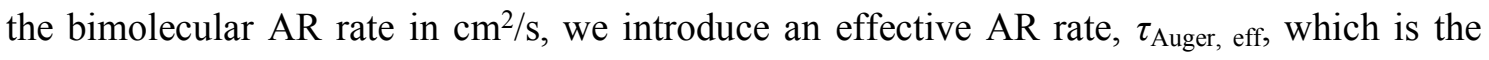
instantaneous rate that can be approximated by using the initial exciton density $\tilde{n}(0)$, according to $\tau_{\text {Auger,eff }}=1 /[\beta \times \tilde{n}(0)]$. The effective Auger recombination times are plotted against the initial exciton density for the four NPL samples in Figure S5 in the SI. It should be noted that $\tau_{\text {Auger,eff }}$ calculated from $\beta$ using Equation 2 cannot be uniquely identified with a specific term in the multiexponential fit. It describes the dynamics occurring over a broad range, from tens to hundreds of picosecond, that are described separately by $\tau_{1}$ and $\tau_{2}$ in the case of the multiexponential fit.

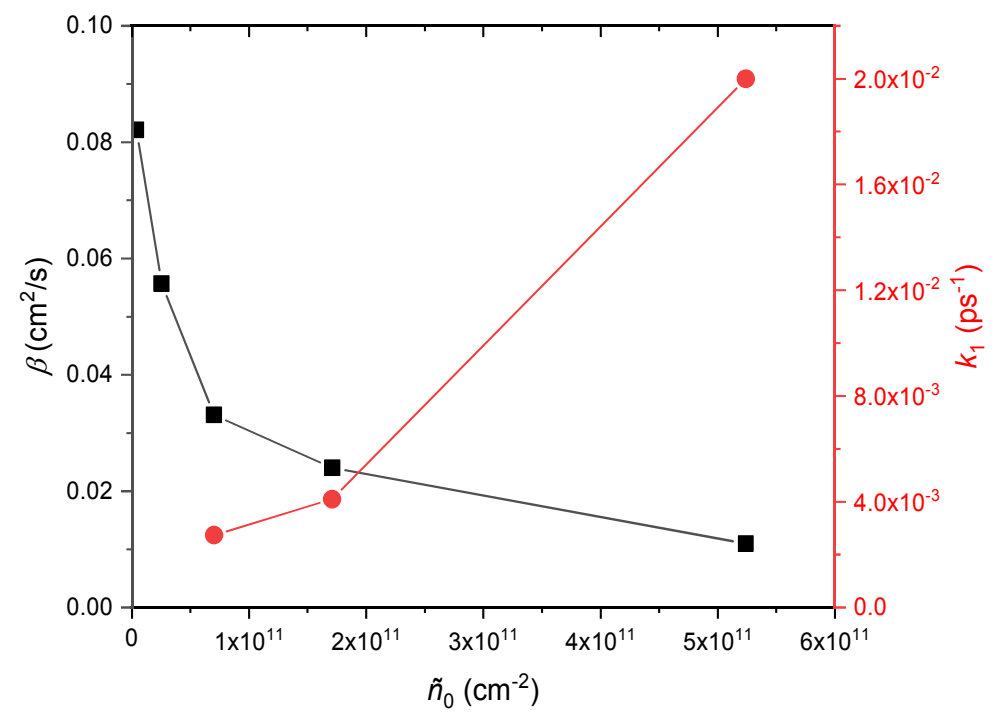

Figure 3. Evolution of the effective bimolecular AR rate $\beta$ (black squares) and of the effective linear AR rate constant $k_{1}$ (red dots) with the initial exciton density extracted from the TR-PL 
decay fit of MAPI $n=3$ NPLs excited at $400 \mathrm{~nm}$. While $\beta$ decreases with $\tilde{n}_{0}$, the corresponding effective linear AR time $\tau_{\text {Auger,eff }}=1 /\left[\beta \times \tilde{n}(0)\right.$ follows the same behavior than $\tau_{1}=1 / k_{1}$ (see Figure S5 in the SI).

For moderate exciton fluence, the results in Figure $\mathbf{3}$ clearly show a diffusion-limited excitonexciton annihilation process with decreasing bimolecular AR rate and increasing corresponding linear rate with $\tilde{n}_{0}$. Furthermore, the values of the bimolecular AR rates in the order of $10^{-2}$ $\mathrm{cm}^{2} / \mathrm{s}$ are consistent with the exciton diffusion constant reported by Prins and $c o$. in the case of 2D lead iodide perovskite thin film based on butylammonium ligands. ${ }^{40}$ From the trend of the effective linear and bimolecular AR rates, we found it more appropriate to use the multiexponential fit, clearly showing faster dynamics occurring at larger initial exciton density. We further note that the AR time is increasing in NPL samples with larger dimensionality: MAPI $n=3$ NPLs seem to present a much-reduced effective linear AR rate than FAPI and MAPI $n=2$ (Figure S5). Moreover, CsPI $n=2$ NPLs presenting a lateral confinement show the fastest AR dynamics at low exciton density, i.e. this latter sample displays an effective linear rate that is less diffusion-limited. We can conclude from these results that the diffusion constant is higher, or the exciton wavefunction delocalization in the $2 \mathrm{D}$ plane is larger, in more strongly-confined NPLs.

At higher excitation fluences, $\tau_{1}$ and the effective AR lifetimes match. However, they attain asymptotically a limiting value that is comparable to the temporal resolution of our TCSPC setup (IRF about $60 \mathrm{ps)}$ ). To determine the intrinsic AR rate (i.e. not diffusion-limited), we need to access the high fluence dynamics with a much higher temporal resolution.

Visible femtosecond transient absorption (TA). TA is a spectroscopic technique providing a much higher temporal resolution than TCSPC. Excitation fluence-dependent TA experiments were carried out on the four NPL samples using band-edge excitation to avoid the effect of cooling in the early time dynamics. A detailed description of our TA setup with pump energy 
tunable with a visible non-collinear optical parametric amplifier (NOPA) is given in the EXPERIMENTAL SECTION. The NPLs were refreshed within the cuvette using a peristaltic pump with adapted tubing to prevent from photo-degradation and photo-charging effects. The transient absorption signal $\Delta \mathrm{A}$ we focus on in this study corresponds to the photo-induced bleach (PIB) coming from the saturation of the band-edge transition due to the presence of excitons generated by the pump in the lowest energy levels. ${ }^{32}$

From a global fit analysis of the TA data, we found that the line-shapes where conserved within the time-range of interest (ps-ns), including no significant spectral shift in the PIB of the bandedge transition (Figure S6 in SI). Consequently, the kinetic trace at the PIB maximum averaged over a few nanometers can be fitted directly. The sign of the negative PIB signal was inverted in order to facilite comparisons with TR-PL analysis and the PIB decays were normalized at long time to highlight the extra faster decay component appearing at high excitation fluence. The data are shown in Figure 4a for MAPI $n=3$ NPLs and Figure S7 in the SI for the three $n=2$ NPL samples. Similarly with TR-PL dynamics, the TA decays were fit by a multiexponential function. The resulting fit parameters are given in Table S7-S10 in the SI.

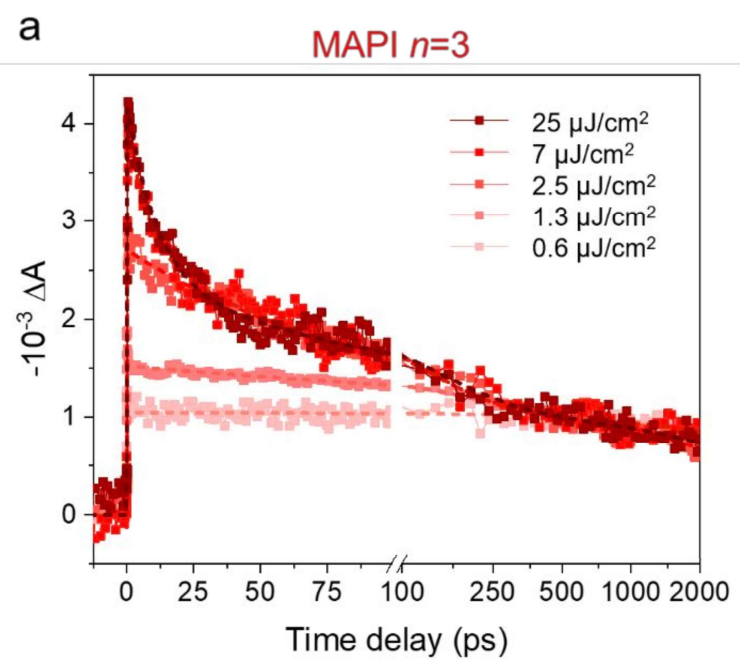

b

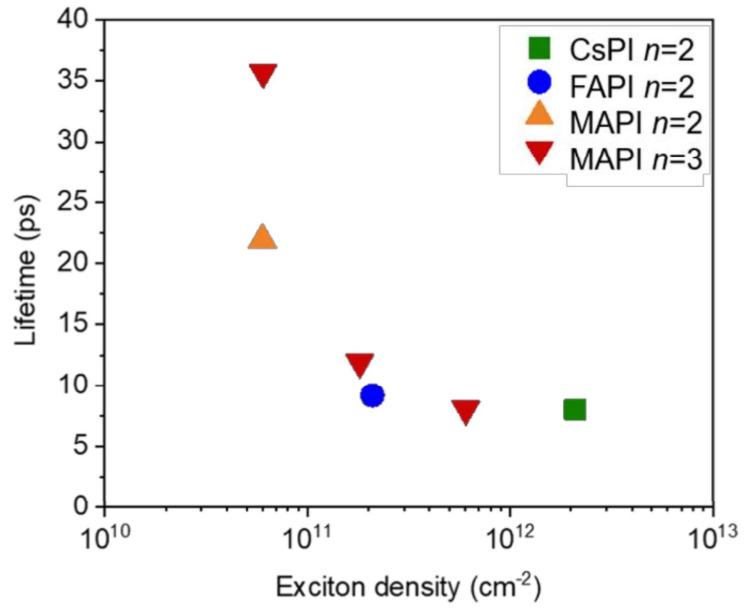


Figure 4. (a) Band-edge PIB traces of MAPI $n=3$ NPLs normalized at long time for different excitation fluences (squares) and multiexponential fit (dashed lines). (b) Early-time AR time constants $\tau_{1}$ extracted from the TA data of MAPI $n=3$ NPLs at three different fluences and in the highest excitation fluence TA decay traces of MAPI, FAPI, and CsPI $n=2$ NPLs.

One kinetic component in the nanosecond time range was enough to fit the traces measured at the lowest excitation fluences (about $0.6-0.7 \mu \mathrm{J} / \mathrm{cm}^{2}$ ), which is in agreement with TR-PL data (see the calculation of the number of excitons and exciton density in Table S11 in the SI). The long-time constants are consistent with the nanosecond single-exciton recombination times measured in TR-PL for all the samples, even if the total TA time range is much shorter (about $2 \mathrm{~ns}$ compared to $12 \mathrm{~ns}$ for TR-PL). Increasing the excitation fluence to of few $\mu \mathrm{J} / \mathrm{cm}^{2}$ leads to the appearance of additional faster kinetic components, a first on the order of a few hundreds of ps, and a second as short as sub-10 ps. Both time constants decrease with the excitation fluence. In line with the discussion of the TR-PL data, we assign these faster components to multiexciton processes, notably Auger recombination. However, the very fast $(<60 \mathrm{ps})$ dynamics could not be determined by TR-PL due to its limited IRF. The short time constants $\left(\tau_{1}\right)$ obtained by TA are plotted as a function of the exciton density for the four samples in Figure $4 \mathbf{b}$. The isolated $\tau_{1}$ components tend to the same value for all the measured NPL samples, corresponding to an intrinsic Auger recombination time (not diffusion-limited). This shows that the AR process can be described by a collision process (or a short-range energy transfer) without involving a recombination probability per collision. We further note that this sub-10 ps component is much faster compared to other nanomaterials such as II-VI and III-V semiconductors that typically have an AR time on the order of a few hundreds of picoseconds. The second time component $\left(\tau_{2}\right)$ on the order of hundreds of ps is assigned to a diffusion-limited Auger recombination process. 
To further address how the multiexciton recombination rate depends on the exciton density in the two-dimensional perovskite structures, the corresponding inter-exciton distance $d_{\mathrm{X}-\mathrm{X}}$ can be calculated. In the case of MAPI $n=3$ NPLs, an additional component with a time constant on the order of hundreds of ps appears at excitation fluences as low as $1.3 \mu \mathrm{J} / \mathrm{cm}^{2}$, corresponding to an initial exciton density of $3 \times 10^{10} \mathrm{~cm}^{-2}$ (calculation in the SI). This leads to an average inter-exciton distance $d_{\mathrm{x}-\mathrm{x}} \approx 57 \mathrm{~nm}$ (center to center distance, see SI). In the same manner, we estimated the exciton-exciton distance from the excitation fluence where an ultrafast component as short as 10 ps becomes predominant ( $>40 \%$ in amplitude at $25 \pm 4 \mu \mathrm{J} / \mathrm{cm}^{2}$ ). For this initial exciton density of $(6 \pm 2) \times 10^{11} \mathrm{~cm}^{-2}, d_{\mathrm{x}-\mathrm{x}} \approx 13 \mathrm{~nm}$. For this distance only four to six times larger than the exciton Bohr radius $a_{\mathrm{B}}$ reported for $\mathrm{MAPbI}_{3}$ perovskite $\left(a_{\mathrm{B}}<3 \mathrm{~nm}^{41}\right)$, the excitons must overlap spatially as they recombine efficiently. Assuming an isotropic exciton delocalization in the $2 \mathrm{D}$ plane, we can estimate the coherent exciton radius $a_{\mathrm{x}}$ (i.e. the characteristic size of the extent of delocalization of the exciton center-of-mass motion ${ }^{18}$ to be about $d_{\mathrm{x}-\mathrm{x}, \min } / 2=6.5 \mathrm{~nm}$. For $d_{\mathrm{x}-\mathrm{x}}$ much larger than the delocalization length, we can only assume that the multiexciton AR rate is limited by the exciton diffusion in the $2 \mathrm{D}$ plane. In that case, the effective AR time depends strongly on the sample geometry. This could explain the range of $\mathrm{AR}$ rates reported by $\mathrm{Li}$ et al. in 5 ML-thick $\mathrm{CsPbBr}_{3} \mathrm{NPLs}$ with different lateral sizes, without the need of invoking a different AR probability per exciton collision. ${ }^{12}$ It also means that the mono-exponential decay usually extracted for discrete biexcitonic to mono-excitonic recombination process in semiconductor nanostructures is not the intrinsic biexcitonic rate in anisotropic perovskite samples such as 2D NPLs. The intrinsic AR rate can only be obtained at high excitation fluence where the initial photogenerated excitons overlap before diffusion takes place (reaction-limited AR).

Multiple exciton generation in 2D NPLs. So far, the exciton-exciton interactions were probed at high excitation fluences, where each absorbed photon creates a unique electron-hole pair and 
multiple excitons within a single NPL decay predominantly through the Auger recombination process. We now investigate the case of high photon energy, which can potentially lead to multiexciton generation, even in the case of low excitation fluence.
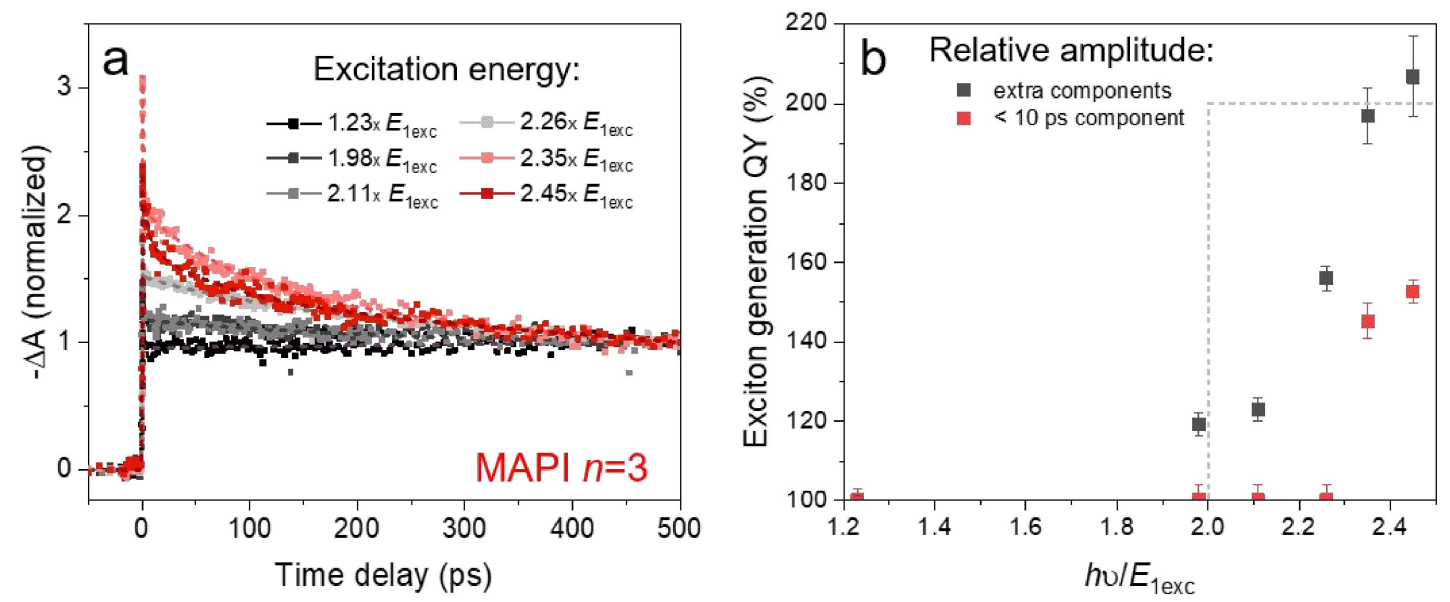

Figure 5. (a) PIB traces at low fluence of MAPI $n=3$ NPLs (band-edge detection, normalized at $500 \mathrm{ps)}$ for different excitation photon energies (corresponding to 500, 310, 290, 270, 260 and $250 \mathrm{~nm}$ in wavelength). Experimental data are represented by squares, multi-exponential fits are represented by dashed lines. (b) Evolution of the amplitude of the early-time component relative to the single exciton one in MAPI $n=3$ NPLs, including the sub- 10 to hundreds of ps components (black squares) and sub-10 ps only (red squares). Dashed grey lines represent the theoretical limit of the maximum quantum yield of generated excitons, $200 \%$, with an excitation threshold of $2 \times \mathrm{E}_{1 \text { exc }}$.

We focused the analysis on MAPI $n=3$ NPLs which present the lowest optical bandgap energy $\left(E_{1 \mathrm{exc}} \approx 1.95 \mathrm{eV}\right)$ among the four synthesized samples. This allows us to excite the NPLs at various energies $E$ in the ultraviolet (UV), using the second harmonic of the NOPA, well-above (about $2.45 \times E_{\text {lexc }}, 250 \mathrm{~nm}$ ) and below $(e . g$. about $320 \mathrm{~nm})$ the minimum required threshold for MEG $\left(E=2 \times E_{1 \text { exc. }}\right)$ To avoid multiphoton absorption within a single NPLs as far as possible, the excitation pulse energy was decreased to below $1 \mathrm{~nJ}$, while keeping well-behaved 
TA dynamics $\left(\triangle \mathrm{A}\right.$ from PIB signal around $\left.0.5 \times 10^{-3}\right)$. The corresponding exciton density for $250 \mathrm{~nm}$ excitation was for example calculated to be less than $10^{11} \mathrm{~cm}^{-2}$ (see SI with the full absorption spectrum of the sample Figure S8). The extracted PIB dynamics measured at the band-edge and normalized at long times are displayed in Figure 5a for six different excitation wavelengths. The corresponding fit parameters are in the Table S12 in SI.

While for a visible excitation the PIB dynamics can be well described by a mono-exponential decay with a long-time component of about 10 ns (represented in black in Figure 5a, excitation at $1.23 \times E_{\text {lex }}$ corresponding to $500 \mathrm{~nm}$ ), extra decay components with time constants ranging between few and hundreds of ps emerge for UV excitation. We also observe a coherent artifact at times below $300 \mathrm{fs}$. This is taken into account in the exponential fit but not considered any further. Similar to the previous analysis, the faster components can be isolated from the longtime single-exciton dynamics after normalization at long times (Figure S9 in SI). This faster dynamics could be interpreted as the indirect observation of MEG, since the excitation fluence was reduced to a minimum while exciting the NPLs with high-energy photons. We should note that here, per opposition with visible TA experiments, we expected at most two excitons per NPL (for low enough excitation fluence). The component characterized by a time constant on the order of hundreds of ps is directly observed at relatively low energy excitation $\left(1.98 \times E_{\text {lexc }}\right.$ and $2.11 \times E_{1 \text { exc }}$ ) with a relatively low amplitude. However, it becomes much more pronounced starting from $2.26 \times E_{\text {lexc }}(270 \mathrm{~nm}$ in wavelength $)$. At $E \geq 2.35 \times E_{\text {lexc }}$, an additional component of a few picoseconds appears. It should be kept in mind that this time constant is much longer than the temporal resolution ( $<300$ fs for UV excitation).

The sum of amplitudes of the extra dynamics relative to the long one can be plotted versus the excitation energy relative to the optical bandgap one $\left(h v / E_{\text {lexc }}\right)$. This leads to an apparent number of excitons generated by a single photon (exciton generation quantum yield, QY), seen in Figure 5b (black squares), as previously reported for perovskite nanocrystals ${ }^{14,19}$ and other 
semiconductor nanostructures. ${ }^{42-44}$ From this plot, it seems that more than one exciton starts to be generated just below the theoretical threshold $\left(1.98 \times E_{\text {lexc }}\right)$ and the maximum MEG efficiency $(200 \%)$ is reached at $2.45 \times E_{1 \mathrm{exc}}$. In the following, we argue that only the fastest component of a few picoseconds corresponds to the indirect observation of MEG through the resulting fast Auger recombination (red squares Figure 5b), while the intermediate component with a time constant of hundreds of picoseconds arises from side effects of high excitation photon energy.

First, several experiments were performed to check that the short picosecond component did not arise from a solvent effect. The reason is that toluene starts to absorb light below $290 \mathrm{~nm}$. Attempts to re-disperse the $n=3$ NPLs or to synthesize them directly in chloroform $\left(\mathrm{CHCl}_{3}\right)$ solvent with negligible absorption in the whole UV range of interest, were unsuccessful. Nevertheless, liquid scintillation experiments reveal that no emission could be detected from the perovskite NPLs after excitation of the toluene molecules, showing the absence of efficient energy transfer (see discussion in the SI). Furthermore, the direct synthesis of thinner MAPI $n=2$ NPLs in chloroform was possible and TA measurements on this sample do not show any picosecond component when exciting at $250 \mathrm{~nm}$, even at higher excitation fluence (Figure S10a in SI). From our point of view, the MEG threshold was not reached in these materials excited under similar conditions to those used for MAPI $n=3$ NPLs, since they have a higher optical bandgap.

Instead, these NPLs show an intermediate component characterized by a time constant on the order of hundreds of ps after excitation at $250 \mathrm{~nm}$, but having a much-reduced amplitude compared to the same NPLs dispersed in toluene (Figure S10a in SI). We interpret this result by the enhanced stability of the NPLs in chloroform versus toluene, which leads to a reduction of the intermediate component due to non-radiative pathways induced at high photon energy exposition. Similarly to the NPL $n=3$ sample in toluene, we found a much higher proportion of 
this intermediate component when exciting at $250 \mathrm{~nm}$ compared to $310 \mathrm{~nm}$ (Figure $\mathbf{S 1 0 b}$ in SI). If this component was due to MEG, it would lead to a QY of about $134 \%$ when excited at $250 \mathrm{~nm}$ (corresponding to an energy of $2.28 \times E_{1 \mathrm{exc}}$ ).

Reported observations of MEG in colloidal semiconductor nanostructures have often been controversial. That is essentially because the MEG process is not directly observed and the measurements are subject to artefacts, such as photo-charging effects leading to other Auger recombination process (trion-related). ${ }^{5}$ In addition, experiments should be performed as substantially lower excitation fluence to avoid the direct generation of multiple excitons via multiphoton absorption. Here, the smooth evolution between 260 and $270 \mathrm{~nm}$ of the toluene absorption (Figure S8) and of the MAPI $n=3$ NPL absorption cross section, cannot explain why the few picosecond component is only observed at $260 \mathrm{~nm}$ under similar excitation condition. In perovskite NPLs, the strong dependence of the AR rate on the average inter-exciton distance due to the limited exciton delocalization length allows us to unambiguously rule out the possibility that the short ps component could result from an excessive excitation fluence. Indeed, even if the low excitation fluencies used here can still generate more than one exciton per NPL (assuming a Poisson distribution ${ }^{16}$ ), the resulting Auger recombination rate will be at least one order of magnitude slower (diffusion-limited AR, see above). The calculated exciton density obtained for the $250 \mathrm{~nm}$ excitation of MAPI $n=3$ NPLs is found to be $10^{11} \mathrm{~cm}^{-2}$ at maximum (see SI), which is an order of magnitude lower than the density needed to obtained the sub-10 ps component in the case of one photon generates a single exciton. On the contrary, multiple excitons photogenerated from a single high-energy photon will be spatially very close, resulting in a diffusion-less AR time, in the picosecond time range. This is similar to what was measured for high excitation fluence in the visible, when the coherent delocalization areas of two near-lying excitons overlap ( $>25 \mu \mathrm{J} / \mathrm{cm}^{2}$ ). The Auger recombination induced by MEG is a reaction of "geminate" biexciton formation (i.e. generated directly or indirectly from the same 
photon, depending on the mechanism ${ }^{45}$ ), while at high excitation density, the Auger process is due to recombination of excitons generated by the absorption of different photons at distinct locations. For nanostructures with a highly delocalized center-of-mass motion wavefunction, no clear distinction can be made between the two different origins of the Auger recombination. Here, the limited exciton delocalization length in the 2D plane of the perovskite NPLs and their highly anisotropic shape allows to isolate the contribution of MEG-induced AR by its characteristically fast decay rate. Finally, we can notice that the measured threshold for MEG in $n=3$ NPLs is well above the theoretical limit of $2 \times E_{1 \text { exc }}$. This can explain why no biexciton generation could be observed in the $n=2$ NPL samples with larger confinement effects.

\section{CONCLUSIONS}

The multiple exciton recombination in strongly-confined 2D perovskite NPLs is dominated by non-radiative Auger recombination. Due to the large asymmetric geometry of these samples and the limited exciton wavefunction delocalization, the Auger recombination rate depends strongly on the excitation fluence. At low fluence, when the initial average inter-exciton distance is several tens of nanometers, the Auger recombination is limited by exciton diffusion and occurs on a timescale of several tens to hundreds of picoseconds. In contrast, high excitation fluences produce "overlapping" excitons with inter-exciton distances only a few times the exciton Bohr radius, resulting in recombination times of less than 10 ps. This fast rate can explain why no efficient lasing could be obtained in these 2D materials. Finally, the dependence of the AR rate on the inter-exciton distance allows us to identify the multiexciton dynamics resulting from the multiple exciton generation process occurring at high photon energy excitation (in the ultraviolet range). For the first time we report the energy threshold of this multiple exciton generation in colloidal 2D perovskite NPLs. These findings will hopefully contribute to the ongoing research and development of light-emitting devices using 2D 
perovskite structures. In particular, the fast AR rates and efficient MEG make the NPLs promising for applications in single photon sources and photodetectors, respectively.

\section{ASSOCIATED CONTENT}

Supporting Information. This includes the synthesis and characterization of the NPL samples, additional TR-PL and TA data, table of the fitting parameters, details of the calculation of the exciton density, discussion on the time scale of the radiative biexciton rate and on the liquid scintillation experiments. The following files are available free of charge.

\section{AUTHOR INFORMATION}

\section{Corresponding Author}

*Corresponding author: elsa.cassette@,cea.fr

\section{ORCID}

Carolina Villamil Franco: 0000-0002-8738-9842

Benoît Mahler: 0000-0001-5471-5608

Thomas Gustavsson : 0000-0001-7030-6812

Elsa Cassette: 0000-0002-1471-6558

\section{Notes}

The authors declare no competing financial interest.

\section{Author Contributions}


The manuscript was written through contributions of all authors. All authors have

given approval to the final version of the manuscript.

\section{Funding Sources}

This work was supported by the French Agence Nationale de la Recherche (grant ANR-16ACHN-0022-01).

\section{ACKNOWLEDGMENT}

E.C. thanks O. Gobert and F. Lepetit for their assistance in the optical and electronic development of the TA apparatus. C.V.F. and E.C. thank S. Guizard and D. Markovitsi for scientific discussion. C.V.F. and E.C. thank Philippe Cassette for the liquid scintillation experiments performed at the LNHB in CEA Saclay. E.C. thanks the ANR program ACHN for funding the CaMPUUS project. C.V.F thanks the University Paris-Saclay for the ED 2MIB Scholarship. B.M. thanks the Université de Lyon PALSE program (ANR-11-IDEX-0007) and C.C. thanks the LabEx PALM of Paris-Saclay University (ANR-10-LABX-0039-PALM), both overseen by the ANR under the framework "Investissements d'avenir".

\section{ABBREVIATIONS}

$\mathrm{AR}$, Auger recombination; $\mathrm{BBO}, \mathrm{Ba}\left(\mathrm{BO}_{2}\right)_{2} ; \mathrm{CCD}$, charge coupled device; CsPI, cesium lead iodide; CW, continuous-wave; FAPI, formamidinium lead iodide; IRF, instrument response function; LARP, ligand assisted re-precipitation; LED, electroluminescent diode; MAPI, methylammonium lead iodide; MEG, multiple exciton generation; ML, monolayer; NC, nanocrystal; NOPA, non-collinear optical parametric amplifier; NPL, nanoplatelet; OD, optical density; PIB, photo-induced bleach; QY, quantum yield; TA, transient absorption; TCSPC, 
1

2

3

4

5

6

7

8

9

10

11

12

13

14

15

16

17

18

19

20

21

22

23

24

25

26

27

28

29

30

31

32

33

34

35

36

37

38

39

40

41

42

43

44

45

46

47

48

49

50

51

52

53

54

55

56

57

58

59

60

time-correlated single photon counting; TR-PL, time-resolved photoluminescence; UV, ultraviolet; WL, white-light; 2D, two-dimensional. 
TOC

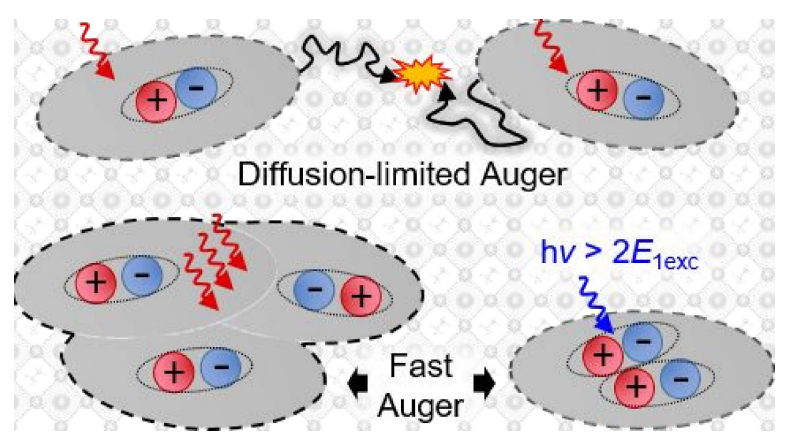

\section{REFERENCES}

(1) Fu, Y.; Zhu, H.; Chen, J.; Hautzinger, M. P.; Zhu, X. Y.; Jin, S. Metal Halide

Perovskite Nanostructures for Optoelectronic Applications and the Study of Physical Properties. Nature Reviews Materials. Nature Publishing Group March 2019, pp 169188.

(2) Zhang, Q.; Su, R.; Du, W.; Liu, X.; Zhao, L.; Ha, S. T.; Xiong, Q. Advances in Small Perovskite-Based Lasers. Small Methods 2017, 1 (9), 1700163.

(3) Zhu, H.; Fu, Y.; Meng, F.; Wu, X.; Gong, Z.; Ding, Q.; Gustafsson, M. V.; Trinh, M. T.; Jin, S.; Zhu, X. Lead Halide Perovskite Nanowire Lasers with Low Lasing Thresholds and High Quality Factors. Nat. Mater. 2015, 14 (6), 636-642.

(4) She, C.; Fedin, I.; Dolzhnikov, D. S.; Demortière, A.; Schaller, R. D.; Pelton, M.; Talapin, D. V. Low-Threshold Stimulated Emission Using Colloidal Quantum Wells. Nano Lett. 2014, 14 (5), 2772-2777.

(5) Klimov, V. I. Multicarrier Interactions in Semiconductor Nanocrystals in Relation to the Phenomena of Auger Recombination and Carrier Multiplication. Annu. Rev. Condens. Matter Phys. 2014, 5 (1), 285-316.

(6) Klimov, V. I. Spectral and Dynamical Properties of Multiexcitons in Semiconductor 
Nanocrystals. Annu. Rev. Phys. Chem. 2007, 58 (1), 635-673.

(7) Makarov, N. S.; Guo, S.; Isaienko, O.; Liu, W.; Robel, I.; Klimov, V. I. Spectral and Dynamical Properties of Single Excitons, Biexcitons, and Trions in Cesium-LeadHalide Perovskite Quantum Dots. Nano Lett. 2016, 16 (4), 2349-2362.

(8) Castañeda, J. A.; Nagamine, G.; Yassitepe, E.; Bonato, L. G.; Voznyy, O.; Hoogland, S.; Nogueira, A. F.; Sargent, E. H.; Cruz, C. H. B.; Padilha, L. A. Efficient Biexciton Interaction in Perovskite Quantum Dots Under Weak and Strong Confinement. ACS Nano 2016, 10 (9), 8603-8609.

(9) Fang, H.-H.; Protesescu, L.; Balazs, D. M.; Adjokatse, S.; Kovalenko, M. V.; Loi, M. A. Exciton Recombination in Formamidinium Lead Triiodide: Nanocrystals versus Thin Films. Small 2017, 13 (32), 1700673.

(10) Delport, G.; Chehade, G.; Lédeé, F.; Diab, H.; Milesi-Brault, C.; Trippé-Allard, G.; Even, J.; Lauret, J. S.; Deleporte, E.; Garrot, D. Exciton-Exciton Annihilation in TwoDimensional Halide Perovskites at Room Temperature. J. Phys. Chem. Lett. 2019, 10 (17), 5153-5159.

(11) Deng, S.; Shi, E.; Yuan, L.; Jin, L.; Dou, L.; Huang, L. Long-Range Exciton Transport and Slow Annihilation in Two-Dimensional Hybrid Perovskites. Nat. Commun. 2020, $11(1), 1-8$.

(12) Li, Q.; Yang, Y.; Que, W.; Lian, T. Size- and Morphology-Dependent Auger Recombination in $\mathrm{CsPbBr}_{3}$ Perovskite Two-Dimensional Nanoplatelets and OneDimensional Nanorods. Nano Lett. 2019, 19 (8), 5620-5627.

(13) Vale, B. R. C.; Socie, E.; Burgos-Caminal, A.; Bettini, J.; Schiavon, M. A.; Moser, J. E. Exciton, Biexciton, and Hot Exciton Dynamics in $\mathrm{CsPbBr} 3$ Colloidal Nanoplatelets. 
J. Phys. Chem. Lett. 2020, 387-394.

(14) Cong, M.; Yang, B.; Chen, J.; Hong, F.; Yang, S.; Deng, W.; Han, K. Carrier Multiplication and Hot-Carrier Cooling Dynamics in Quantum-Confined CsPbI3 Perovskite Nanocrystals. J. Phys. Chem. Lett. 2020, 11 (5), 1921-1926.

(15) Pelant, I.; Valenta, J. Luminescence Spectroscopy of Semiconductors; Oxford University Press: Oxford, 2012; Vol. 9780199588.

(16) Klimov, V. I. Nanocrystal Quantum Dots -2nd Edition; 2010.

(17) Klimov, V. I.; McGuire, J. A.; Schaller, R. D.; Rupasov, V. I. Scaling of Multiexciton Lifetimes in Semiconductor Nanocrystals. Phys. Rev. B - Condens. Matter Mater. Phys. 2008, $77(19), 195324$.

(18) Li, Q.; Lian, T. Exciton Spatial Coherence and Optical Gain in Colloidal TwoDimensional Cadmium Chalcogenide Nanoplatelets. Acc. Chem. Res. 2019, 52 (9), 2684-2693.

(19) Li, M.; Begum, R.; Fu, J.; Xu, Q.; Koh, T. M.; Veldhuis, S. A.; Grätzel, M.; Mathews, N.; Mhaisalkar, S.; Sum, T. C. Low Threshold and Efficient Multiple Exciton Generation in Halide Perovskite Nanocrystals. Nat. Commun. 2018, 9 (1), 3-9.

(20) de Weerd, C.; Gomez, L.; Capretti, A.; Lebrun, D. M.; Matsubara, E.; Lin, J.; Ashida, M.; Spoor, F. C. M.; Siebbeles, L. D. A.; Houtepen, A. J.; Suenaga, K.; Fujiwara, Y.; Gregorkiewicz, T. Efficient Carrier Multiplication in $\mathrm{CsPb}_{3}$ Perovskite Nanocrystals. Nat. Commun. 2018, 9 (1), 1-3.

(21) Smith, C.; Binks, D. J. Multiple Exciton Generation in Colloidal Nanocrystals. Nanomaterials 2014, 4, 19-45.

(22) Weidman, M. C.; Goodman, A. J.; Tisdale, W. A. Colloidal Halide Perovskite 
Nanoplatelets: An Exciting New Class of Semiconductor Nanomaterials. Chem. Mater. 2017, 29 (12), 5019-5030.

(23) Hintermayr, V. A.; Polavarapu, L.; Urban, A. S.; Feldmann, J. Accelerated Carrier Relaxation through Reduced Coulomb Screening in Two-Dimensional Halide Perovskite Nanoplatelets. ACS Nano 2018, 12 (10), 10151-10158.

(24) Levchuk, I.; Osvet, A.; Tang, X.; Brandl, M.; Perea, J. D.; Hoegl, F.; Matt, G. J.; Hock, R.; Batentschuk, M.; Brabec, C. J. Brightly Luminescent and Color-Tunable Formamidinium Lead Halide Perovskite $\mathrm{FAPbX}_{3}(\mathrm{X}=\mathrm{Cl}, \mathrm{Br}, \mathrm{I})$ Colloidal Nanocrystals. Nano Lett. 2017, 17 (5), 2765-2770.

(25) Weidman, M. C.; Seitz, M.; Stranks, S. D.; Tisdale, W. A. Highly Tunable Colloidal Perovskite Nanoplatelets through Variable Cation, Metal, and Halide Composition. ACS Nano 2016, 10 (8), 7830-7839.

Blancon, J. C.; Tsai, H.; Nie, W.; Stoumpos, C. C.; Pedesseau, L.; Katan, C.;

Kepenekian, M.; Soe, C. M. M.; Appavoo, K.; Sfeir, M. Y.; Tretiak, S.; Ajayan, P. M.; Kanatzidis, M. G.; Even, J.; Crochet, J. J.; Mohite, A. D. Extremely Efficient Internal Exciton Dissociation through Edge States in Layered 2D Perovskites. Science 2017, 355 (6331), 1288-1292.

(27) Paritmongkol, W.; Dahod, N. S.; Stollmann, A.; Mao, N.; Settens, C.; Zheng, S. L.; Tisdale, W. A. Synthetic Variation and Structural Trends in Layered Two-Dimensional Alkylammonium Lead Halide Perovskites. Chem. Mater. 2019, 31 (15), 5592-5607.

(28) Eperon, G. E.; Stranks, S. D.; Menelaou, C.; Johnston, M. B.; Herz, L. M.; Snaith, H. J. Formamidinium Lead Trihalide: A Broadly Tunable Perovskite for Efficient Planar Heterojunction Solar Cells. Energy Environ. Sci. 2014, 7 (3), 982-988. 
(29) Baghani, E.; O’Leary, S. K.; Fedin, I.; Talapin, D. V.; Pelton, M. Auger-Limited Carrier Recombination and Relaxation in CdSe Colloidal Quantum Wells. J. Phys. Chem. Lett. 2015, 150302175751004.

(30) Johnston, M. B.; Herz, L. M. Hybrid Perovskites for Photovoltaics: Charge-Carrier Recombination, Diffusion, and Radiative Efficiencies. Acc. Chem. Res. 2016, 49 (1), $146-154$.

(31) Eperon, G. E.; Jedlicka, E.; Ginger, D. S. Biexciton Auger Recombination Differs in Hybrid and Inorganic Halide Perovskite Quantum Dots. J. Phys. Chem. Lett. 2018, 9 (1), 104-109.

(32) Klimov, V. I. Optical Nonlinearities and Ultrafast Carrier Dynamics in Semiconductor Nanocrystals. J. Phys. Chem. B 2000, 104 (26), 6112-6123.

(33) Makarov, N. S.; Guo, S.; Isaienko, O.; Liu, W.; Robel, I.; Klimov, V. I. Spectral and Dynamical Properties of Single Excitons, Biexcitons, and Trions in Cesium-LeadHalide Perovskite Quantum Dots. Nano Lett. 2016, 16 (4), 2349-2362.

(34) Binks, D. J. Multiple Exciton Generation in Nanocrystal Quantum Dots - Controversy, Current Status and Future Prospects. Phys. Chem. Chem. Phys. 2011, 13 (28), 1269312704.

(35) Lakowicz, J. R. Principles of Fluorescence Spectroscopy; Springer, 2006.

(36) Kirm, M.; Nagirnyi, V.; Feldbach, E.; De Grazia, M.; Carré, B.; Merdji, H.; Guizard, S.; Geoffroy, G.; Gaudin, J.; Fedorov, N.; Martin, P.; Vasil'ev, A.; Belsky, A. ExcitonExciton Interactions in $\mathrm{CdWO}_{4}$ Irradiated by Intense Femtosecond Vacuum Ultraviolet Pulses. Phys. Rev. B - Condens. Matter Mater. Phys. 2009, 79 (23), 233103.

(37) Kunneman, L. T.; Tessier, M. D.; Heuclin, H.; Dubertret, B.; Aulin, Y. V.; Grozema, F. 
C.; Schins, J. M.; Siebbeles, L. D. A. Bimolecular Auger Recombination of ElectronHole Pairs in Two-Dimensional CdSe and CdSe/CdZnS Core/Shell Nanoplatelets. $J$. Phys. Chem. Lett. 2013, 4 (21), 3574-3578.

(38) Gösele, U. Anisotropic Diffusion, Long-Range Energy Transfer and Bimolecular Exciton Recombination Kinetics. Chem. Phys. Lett. 1976, 43 (1), 61-64.

(39) Burgel, M.; Van; Wiersma, D. A. The Ultrafast Dynamics of Aggregate Excitons in Water; s.n., 1999.

(40) Seitz, M.; Magdaleno, A. J.; Alcázar-Cano, N.; Meléndez, M.; Lubbers, T. J.;

Walraven, S. W.; Pakdel, S.; Prada, E.; Delgado-Buscalioni, R.; Prins, F. Exciton Diffusion in Two-Dimensional Metal-Halide Perovskites. Nat. Commun. 2020, 11 (1), $1-8$.

(41) Tanaka, K.; Takahashi, T.; Ban, T.; Kondo, T.; Uchida, K.; Miura, N. Comparative Study on the Excitons in Lead-Halide-Based Perovskite-Type Crystals $\mathrm{CH}_{3} \mathrm{NH}_{3} \mathrm{PbBr}_{3}$ $\mathrm{CH}_{3} \mathrm{NH}_{3} \mathrm{PbI}_{3}$. Solid State Commun. 2003, 127 (9-10), 619-623.

(42) Schaller, R. D.; Agranovich, V. M.; Klimov, V. I. High-Efficiency Carrier Multiplication through Direct Photogeneration of Multi-Excitons via Virtual SingleExciton States. Nat. Phys. 2005, 1 (3), 189-194.

(43) Ellingson, R. J.; Beard, M. C.; Johnson, J. C.; Yu, P.; Micic, O. I.; Nozik, A. J.; Shabaev, A.; Efros, A. L. Highly Efficient Multiple Exciton Generation in Colloidal PbSe and PbS Quantum Dots. Nano Lett. 2005, 5 (5), 865-871.

(44) Aerts, M.; Bielewicz, T.; Klinke, C.; Grozema, F. C.; Houtepen, A. J.; Schins, J. M.; Siebbeles, L. D. A. Highly Efficient Carrier Multiplication in PbS Nanosheets. Nat. Commun. 2014, 5, 1-5. 
1

2

3

4

5

6

7

8

9

10

11

12

13

14

15

16

17

18

19

20

21

22

23

24

25

26

27

28

29

30

31

32

33

34

35

36

37

38

39

40

41

42

43

44

45

46

47

48

49

50

51

52

53

54

55

56

57

58

59

60

(45) Shabaev, A.; Hellberg, C. S.; Efros, A. L. Efficiency of Multiexciton Generation in Colloidal Nanostructures. Acc. Chem. Res. 2013, 46 (6), 1242-1251. 